\title{
VON WILLEBRAND FACTOR, C-REACTIVE PROTEIN, NITRIC OXIDE, AND VASCULAR ENDOTHELIAL GROWTH FACTOR IN A DIETARY REVERSAL MODEL OF HYPERCHOLESTEROLEMIA IN RABBIT
}

\author{
Shaghayegh Haghjooyjavanmard ${ }^{\mathrm{a}}$, Mehdi Nematbakhsh ${ }^{\mathrm{a} *}$, Alireza Monajemi ${ }^{\mathrm{a}}$, \\ Masoud Soleimani ${ }^{\mathrm{b}}$
}

\begin{abstract}
a Applied Physiology Research Center, Isfahan University of Medical Sciences, Isfahan, Iran
${ }^{b}$ Hematology Department, School of Medical Sciences, Tarbiat Modares University, Tehran, Iran e-mail: nematbakhsh@gmail.com
\end{abstract}

Received: February 5, 2008; Accepted (with revisions): May 18, 2008

Key words: Endothelial dysfunction/Nitric Oxide/Vascular Endothelial Growth Factor/von Willebrand Factor/C-Reactive Protein/Atherosclerosis

Aims: Endothelial dysfunction is considered a sign of the early vascular changes preceding atherosclerosis. We studied the alteration of von Willebrand Factor (vWF), C - reactive protein (CRP), nitrite and Vascular Endothelial Growth Factor (VEGF) in a dietary reversal model of hypercholesterolemia in rabbit.

Methods: This project was designed in two phases. In phase I, male rabbits $(n=11)$ were fed a $1 \%$ high cholesterol diet for 30 days. Then the diet was replaced with normal rabbit chow for other 30 days (cholesterol withdrawal phase, phase II). To compare the fatty streak formation with normal condition, a control group $(n=6)$ received normal diet during the study. The serum lipid levels, vWF, CRP, nitrite, and VEGF were measured before the experiment and by the end of each phase. Fatty streak formation in the walls of the aortas in both groups (high cholesterol diet and control group) was determined using intima thickness/media thickness (IMT) ratio.

Results: The results indicate that the level of cholesterol, Low Density Lipoproteins (LDL), vWF and CRP increased significantly in phase I, and decreased after hypercholesterolemic diet withdrawal $(p<0.05)$. No statistically significant changes were found in VEGF levels but the serum level of nitrite increased significantly during both phases of the study $(p<0.05)$. The IMT ratio in the walls of aortas was significantly different between the groups in both phases of studies $(\mathrm{p}<0.05)$. There was a significant correlation between nitrite and cholesterol levels in both phases $(\mathrm{r}=0.62$ and $r=0.98, p<0.05)$. Nitrite concentration also correlated with IMT ratio in both phases of the study $(r=0.75$ and $r=-0.99, p<0.05)$. vWF did not correlate with cholesterol but it correlated with IMT ratio in both phases of the study $(r=0.87$ and $r=0.84, p<0.05)$. CRP only correlated with cholesterol in the first phase $(r=0.91, p<0.05)$.

Conclusions: Among the endothelial biomarkers, vWF was found to be a biological marker for identifying the risk of developing atherosclerosis; however a single biomarker may not provide appropriate information.

\section{INTRODUCTION}

Endothelial Dysfunction (ED) is a key variable in the pathogenesis of atherosclerosis and its complications. The presence of ED can be considered a clinical syndrome that is associated with and predicts adverse cardiovascular events ${ }^{1}$. Almost all risk factors that are related to atherosclerosis and cardiovascular disease including hyperlipidemia, hypertension, diabetes, and smoking are associated with ED. The ED is a switch that translates any given risk factor into unfavorable vascular effects. For this reason it is called the "ultimate risk of risk factors" 1 . ED measurement has important implications for cardiovascular disease management, such as primary prevention, evaluating the severity and prognosis of diseases and measuring treatment efficacy ${ }^{2}$.

Several approaches can be used to measure ED. These are categorized into functional assessment of ED (including flow-mediated dilation methods), structural markers of ED (such as IMT ratio) and cellular markers of ED (like circulating endothelial cells and biochemical markers of ED) (ref. ${ }^{2,3}$ ). Of these methods, biomarkers are more practicable in a clinical setting: they are measured in easily obtained blood samples and can be standardized for use in clinical practice ${ }^{4}$.

To date, there is no specific biomarker measurement that can be called a "gold standard" for ED assessment and it is recommended that ED should be investigated in a multifaceted approach ${ }^{5}$.

Indeed, there are several factors which have been proposed as potential markers of endothelial cell activation and injury, and among ED biomarkers, the levels of vWF, CRP, nitrite and VEGF were investigated in this study $^{3-14}$. Elevated vWF is perhaps the most known marker of endothelial injury ${ }^{4,5}$. Raised CRP adds prognostic information on future cardiovascular risk at all levels of the Framingham risk score ${ }^{8,9}$. Nitric Oxide (NO) stable metabolites $^{1-4}$ such as nitrite have been reported to be good markers of endothelial NO production ${ }^{15-17}$ while reduced NO bioactivity is known as an early event in atheroscle- 
rosis ${ }^{15}$. Another biomarker - VEGF is a vascular protective factor and functions as an endogenous regulator of endothelial integrity after injury ${ }^{18}$. It has been shown that VEGF and its receptors are up-regulated in vascular inflammatory and proliferative disorders such as atherosclerosis and restenosis ${ }^{19,20}$.

Biomarkers have been investigated in patients in a number of studies ${ }^{6,7,9,10,13,14}$. However, owing to the longstanding condition of the patients, it is not clear which biomarker changes in the early stage of atherosclerosis. This would provide very valuable information because detection of ED in the early stage of atherosclerosis would offer the chance of reversing the atherogenesis. This study was designed to investigate the effect of hypercholesterolemia as a risk factor of ED on ED biomarkers in a well-known model of atherosclerosis - the hypercholesterolemic rabbit $^{21}$ by measuring vWF, CRP, serum nitrite, and VEGF. These markers were chosen to provide a multifaceted approach to ED.

\section{MATERIALS AND METHODS}

\section{Animals and Experimental design}

The study was reviewed and approved by the Ethics Committee of Isfahan University of Medical Sciences.

Seventeen white male rabbits weighing $2 \pm 0.2 \mathrm{~kg}$ were obtained from the Pasteur Institute of Iran.

After a 1-week acclimatization period and overnight fasting, venous blood samples were taken as pre-experimental sampling to obtain baseline data. Collected blood samples were centrifuged $(10,000 \times g)$, and the resulting supernatant, the serum or plasma, was stored at $-70{ }^{\circ} \mathrm{C}$ until measurement. The plasma was used for vWF measurement and the serum for other biomarkers. The animals were then assigned to two groups. The animals in group HC $(\mathrm{n}=11)$ were fed normal rabbit chow supplemented with $1 \%$ cholesterol. A total of 6 rabbits were on normal chow as control group to investigate fatty streak formation. The normal rabbit chow components were starch $(560 \mathrm{~g} / \mathrm{kg})$, fiber $(280 \mathrm{~g} / \mathrm{kg})$, grass meal $(50 \mathrm{~g} / \mathrm{kg})$, fat mixture $(40 \mathrm{~g} / \mathrm{kg})$, mineral mixture $(39.5 \mathrm{~g} / \mathrm{kg})$, calcium carbonate and bisphosphate $(25 \mathrm{~g} / \mathrm{kg})$ and vitamin mixture $(5.5 \mathrm{~g} / \mathrm{kg}$ ). All animals received $150 \mathrm{~g} /$ day rabbit chow diet and water ad libitum. After 30 days of experiment (phase I), the blood samples were taken and stored again. Five animals from the HC group and three animals from control group were randomly selected, euthanized by an overdose of sodium pentobarbital and ex-sanguinated. The animal's aortas were harvested for pathological investigation. From this point, the hypercholesterolemic diet of the $\mathrm{HC}$ group was terminated and all animals were on a normal diet. The experiment was continued for other 30 days (phase II). At the end of the phase II, the blood sampling and storage were repeated. The rest of the animals were killed and the aortas harvested for pathological investigation.

\section{Lipid and lipoprotein measurements}

Total cholesterol, HDL cholesterol and LDL cholesterol levels were determined using standard enzymatic kits (Pars Azmoon Co, Iran).

\section{Detection of endothelial markers}

VWF (Cedarlane Co, Canada), VEGF (R\&D systems, Minneapolis, USA) and CRP (IBL Co, Germany) were measured using enzyme-linked immuno-sorbent assay kits according to manufacturer's instructions. The serum level of nitrite was measured using a colorimetric assay kit (R\&D Systems, USA) that involves the Griess reaction.

\section{Pathological investigation}

The abdominal aortas were subjected to pathological procedures to verify ED as fatty dots or fatty streak lesions. The entire aorta, from the aortic arch to the external iliac arteries, was dissected out and cleaned of excess adventitious tissue. The aortas were fixed in buffered $10 \%$ formalin for $24 \mathrm{~h}$ and then embedded in paraffin. The paraffin-embedded specimens were sectioned at $5 \mu \mathrm{m}$ (20 sections in succession), stained with haematoxylin and eosin and examined by light microscopy for fatty streaks by two pathologists in a double-blinded manner.

Fatty streak formation was determined by intima thickness and media thickness measurement in all sections. The data were averaged and used to obtain IMT ratio (Intima thickness/Media thickness).

\section{Statistical analysis}

The data are reported as the mean \pm SEM. The statistical software package, SPSS (version 13), was used for the statistical analysis. The data were tested for normality and homogeneity of variance. Paired Student's $t$-test was used to assess the significance of any change before and after treatment, while unpaired Student's $t$-test (equal or unequal variance assumed accordingly) was used to assess the significance of any change between groups. The Spearman's rank correlation test was used to evaluate relationships between variables. The one sample t-test was used for IMT ratio data. Statistical significance was accepted at $p<0.05$.

\section{RESULTS}

\section{Plasma lipids}

The cholesterol-rich diet induced a significant increase in total cholesterol, LDL-cholesterol and HDL-cholesterol within phase I of the study. After hypercholesterolemic diet withdrawal, total plasma cholesterol and LDL reduced significantly but did not return to baseline values within phase II (Table 1).

\section{Endothelial biomarkers}

The serum level of vWF significantly increased by the end of phase I $(0.173 \pm 0.01$ vs. $0.953 \pm 0.2 \mathrm{IU}, \mathrm{p}<0.05)$ and significantly decreased after 30 days of hypercholesterolemic diet withdrawal $(0.195 \pm 0.1 \mathrm{IU}, \mathrm{p}<0.05)$ 
(Table 1). There was no significant difference in vWF levels between baseline and the end of phase II.

The serum level of CRP significantly increased by the end of the first phase $(2.90 \pm 0.08$ vs. $3.22 \pm 0.1 \mu \mathrm{g} / \mathrm{ml}$, $\mathrm{p}<0.05)$ and significantly decreased by the end of the second phase $(2.7 \pm .2 \mu \mathrm{g} / \mathrm{ml}, \mathrm{p}<0.05)$ (Table 1$)$. No significant difference in serum level of CRP was found between baseline and the end of phase II.

There was a trend towards increased serum level of VEGF by the end of phase I ( $202.95 \pm 175.8$ vs. $525.4 \pm 205.2 \mathrm{pmol} / \mathrm{L}, \mathrm{p}=0.13$ ) and also a trend towards decreased serum level of VEGF by the end of phase II $(59.43 \pm 26.6 \mathrm{pmol} / \mathrm{L}, \mathrm{p}=0.1)$ (Table 1). No significant difference in serum level of VEGF was found between baseline and the end of phase II.

We found a trend towards increased serum level of nitrite by the end of the first phase $(8.86 \pm 1.1$ vs. $11.38 \pm 1.8 \mu \mathrm{mol} / \mathrm{L}, \mathrm{p}=0.09)$ and a tendency to increase by the end of the second phase $(19.4 \pm 2.4 \mu \mathrm{mol} / \mathrm{L}$, $\mathrm{p}=0.07)$. At the end of phase II, the serum level of nitrite was significantly different from pre-experiment $(p<0.05)$ (Table 1).

\section{Fatty streak formation}

At the end of phase I, there were no fatty streak lesions in the control group aortas while the IMT ratio was $0.33 \pm 0.1$ in $\mathrm{HC}$ group $(\mathrm{p}=0.07)$. At the end of phase II, the fatty streak lesion formation was significantly higher in the HC group $(0.49 \pm 0.1$ vs. $0, \mathrm{p}<0.05)$.

\section{Endothelial biomarker correlations with cholesterol and fatty streaks in two phases of the study}

There were significant correlations between cholesterol and serum levels of nitrite $(r=0.621, p<0.05)$ and CRP $(r=0.91, p<0.05)$ by the end of the first phase of experiment, but there was no correlation between cholesterol and serums levels of vWF and VEGF in this phase.

In the second phase of the study, only cholesterol had a strong correlation with serum levels of nitrite $(r=0.98$, $\mathrm{p}<0.05)$.

IMT ratio had a strong correlation with $\mathrm{vWF}(\mathrm{r}=0.87$, $\mathrm{p}<0.05)$ and serum nitrite $(\mathrm{r}=0.75, \mathrm{p}<0.05)$ after the first phase of the experiment. In the end of the study, the IMT ratio correlated with $\mathrm{vWF}(\mathrm{r}=84 \%, \mathrm{p}<0.05)$ and with serum level of nitrite $(r=-0.99, p<0.05)$. No statistical correlations were found among other parameters such as NO and VEGF, CRP or vWF.

\section{DISCUSSION}

The cholesterol-rich diet induced a significant increase in total cholesterol, LDL-cholesterol and HDL-cholesterol in the first phase of the study. After hypercholesterolemic diet withdrawal, plasma lipids reduced significantly but did not return to baseline values within 30 days. In this model, the cholesterol-rich diet resulted in the development of early lesions that were representative of fatty streak initiation similar to those in humans. Thus, the model allowed us to examine the initiation of atherosclerosis and risk factor effects after hypercholesterolemic diet withdrawal ${ }^{21,22}$. In a similar model of rabbit hypercholesterolemia with $0.5 \%$ cholesterol-rich diet, the achieved serum level of cholesterol was only one fourth of that observed in our study ${ }^{23}$. This difference may be partly due to higher amount of food administered in the present experiment. Hypercholesterolemia-induced vWF increment found in our study is similar to previous reports ${ }^{4,6,7}$. VWF decreased after cholesterol diet discontinuation (phase II) - a finding that is interesting in the light of previous studies in which treatment with lipid-lowering agents (i.e. fluvastatin or fenofibrate) did not diminish plasma vWF concentration in hyperlipidemic patients ${ }^{4}$. Our results showed that vWF concentration did not correlate with the severity of hypercholesterolemia, which is contrary to the positive correlation between these parameters found in the study by Hernández et al. using a larger sample size of hypercholesterolemic rabbits ${ }^{23}$. Nonetheless, vWF had a strong positive correlation with fatty streak formation in both phases of our study. It is notable that vWF has been reported as a highly relevant biomarker of vascular disease in many other studies ${ }^{3 \cdot 7,23}$.

CRP followed the changes of cholesterol throughout the study - increase after 30 days of hypercholesterolemic

Table 1. The serum levels of total cholesterol, LDL, and HDL, vWF, CRP, Nitrite and VEGF.

\begin{tabular}{|l|c|c|c|}
\hline parameters & baseline & phase I & phase II \\
\hline Cholesterol $(\mathrm{mg} / \mathrm{dl})$ & $117.3 \pm 10.3$ & $2238.37 \pm 132.1^{\#}$ & $1269.52 \pm 115^{\#, *}$ \\
\hline LDL $(\mathrm{mg} / \mathrm{dl})$ & $32.5 \pm 11.5$ & $1259.9 \pm 265.4^{\#}$ & $310.74 \pm 61.2^{\#, *}$ \\
\hline HDL $(\mathrm{mg} / \mathrm{dl})$ & $15.5 \pm 3.2$ & $71.8 \pm 14.9^{\#}$ & $61.21 \pm 8.2^{\#, *}$ \\
\hline vWF $(\mathrm{IU})$ & $0.173 \pm 0.01$ & $0.953 \pm 0.2^{\#}$ & $0.195 \pm 0.1^{*}$ \\
\hline CRP $(\mu \mathrm{g} / \mathrm{ml})$ & $2.90 \pm 0.08$ & $3.22 \pm 0.1^{\#}$ & $2.7 \pm .2^{*}$ \\
\hline Nitrite $(\mu \mathrm{mol} / \mathrm{l})$ & $8.86 \pm 1.1$ & $11.38 \pm 1.8$ & $19.4 \pm 2.4^{\#}$ \\
\hline VEGF $(\mathrm{pmol} / \mathrm{l})$ & $202.95 \pm 175.8$ & $525.4 \pm 205.2$ & $59.43 \pm 26.6$ \\
\hline
\end{tabular}


diet and decrease after the reversal. The former finding is in line with other studies ${ }^{8,9,23,24}$. Concerning the latter, CRP decrement has been reported after statin therapy ${ }^{12}$. In our experiment, elevated plasma CRP correlated with the severity of hypercholesterolemia - a finding that is interesting for it has not been observed in human patients ${ }^{23}$; however it has been shown in another study with hypercholesterolemic rabbits ${ }^{24}$. In any event, the relationship of CRP to total cholesterol and LDL cholesterol remains equivocal and is currently unknown.

We found a trend towards increased VEGF in the first 30 days which decreased after cholesterol withdrawal. Increased plasma VEGF levels in atherosclerosis have been documented also by other authors ${ }^{13,14,18,19}$. Although VEGF release may be a reparative mechanism in the early atherosclerosis ${ }^{20}$, it may also reflect endothelial cell damage. VEGF decrement by lipid-lowering therapy has been demonstrated in patients with hyperlipidemia and atherosclerosis $^{13}$.

The present results indicated that 30 days of cholesterol-feeding (short-term hypercholesterolemia) enhanced the content of nitrite, as has been demonstrated previously $^{26-28}$. Although decreased NO bioactivity has been attributed to ED ( ref. $^{15}$ ) increased plasma level of nitrite has been reported in hypercholesterolemic patients too ${ }^{29}$. Increased level of serum nitrite in our study is different from similar studies with hypercholesterolemic rabbits which reported impaired endothelium-dependent relaxation accompanied by decreased NO production and reduced vessel cyclic guanosine monophosphate (cGMP) production $^{30}$.

It has been suggested that enhanced NO synthesis might be a defense mechanism to compensate for continuous inactivation of $\mathrm{NO}$ and protection against damaging factors ${ }^{29,31}$. The proposed mechanism responsible for the elevation of nitrite may be a significant increase in overall NOS synthesis by other cell types (than endothelium) in advanced lesions composed of the endothelial, neuronal and inducible isoforms of Nitric Oxide Synthase (NOS) enzymes. Despite decreased expression and activity of endothelial NO Synthase (eNOS) which has been indicated in some studies ${ }^{30}$, there are several studies that demonstrated the amount of NOS present in the atherosclerotic vessel wall to be substantially increased compared with normal vessels ${ }^{32-34}$.

Increased NOS mRNA and protein in atherosclerosis is compatible with experiments showing that aortas of hypercholesterolemic rabbits release larger quantities of nitrogen oxides than do normal vessels ${ }^{32}$. Furthermore, using pharmacological probes has suggested that there is an increased production of NO in nonendothelial layers of vessels from hypercholesterolemic animals ${ }^{35}$.

Increased endothelial constitutive NOS Protein and mRNA in rabbit atherosclerotic aorta despite impaired endothelium-dependent vascular relaxation ${ }^{33}$ may be explained by inactivation of NO by oxygen-derived free radicals or masking the increased NO by increased production of endothelium-derived constricting factors.

Another explanation for the discrepancy between the results of these studies may be regional variation in eNOS activity. It has been shown that the eNOS activity in normocholesterolemic rabbits is significantly higher in the downstream regions of aorta compared with the regions located more upstream such as aortic arch and thoracic aorta $^{36}$.

In our study nitrite as a NO metabolite correlated with fatty streak formation in both steps of experiment; however positively correlated in the first phase and negatively correlated in the second phase. Further studies are needed to shed light on the underlying mechanisms.

\section{CONCLUSION}

The aim of the present study was to investigate the impact of early atherosclerosis as a disease state on the known biomarkers of ED. Among these biomarkers vWF, CRP, and VEGF followed risk factor alteration, and nitrite gradually increased during the study along with fatty streaks. Of these markers, vWF and NO significantly correlated with fatty streak formation but only vWF had consistent positive correlation therewith. This study suggests that CRP and VEGF are not significant predictors of atherosclerosis progression. It seems that vWF is the best biological marker for identifying the risk of developing atherosclerosis as a disease state in our study; however it is doubtful that a single biomarker provides appropriate information for all clinical settings associated with other types of risk factors.

Further effort is needed to improve the reliability and reproducibility of these results.

\section{ACKNOWLEDGEMENTS}

This study was supported by Isfahan University of Medical sciences, Isfahan, Iran and Stem Cell Technology CO. Tehran, Iran (Grant \# 384151). Special thanks to Dr. Gheisari, Dr. Mohajeri, F. Mahmoodi, H. Sadeghi, N. Dana and M. Samadi for their valuable helps.

\section{REFERENCES}

1. Bonetti PO, Lerman LO, Lerman A. Endothelial dysfunction: a marker of atherosclerotic risk. Arterioscler Thromb Vasc Biol 2003; 23(2):168-175.

2. Goligorsky MS. Clinical assessment of endothelial dysfunction: combine and rule. Curr Opin Nephrol Hypertens 2006; 15(6):617624.

3. Felmeden DC, Lip GY. Endothelial function and its assessment. Expert Opin Investig Drugs 2005; 14(11):1319-1336.

4. Constans J, Conri C. Circulating markers of endothelial function in cardiovascular disease. Clin Chim Acta 2006; 368(1-2):33-47.

5. Blann AD, Taberner DA. A reliable marker of endothelial cell dysfunction: does it exist? Br J Haematol 1995; 90(2):244-248.

6. Blann AD, Miller JP, McCollum CN. von Willebrand factor and soluble E-selectin in the prediction of cardiovascular disease progression in hyperlipidaemia. Atherosclerosis 1997; 132(2):151156.

7. Blann AD, Davis A, Miller JP, McCollum CN. von Willebrand factor and soluble E-selectin in hyperlipidaemia: relationship to lipids and vascular disease. Am J Hematol 1997; 55(1):15-23. 
8. Pearson TA, Mensah GA, Alexander RW, Anderson JL, Cannon RO, III, Criqui $\mathrm{M}$ et al. Markers of inflammation and cardiovascular disease: application to clinical and public health practice: A statement for healthcare professionals from the Centers for Disease Control and Prevention and the American Heart Association. Circulation 2003; 107(3):499-511.

9. Jialal I, Devaraj S, Venugopal SK. C-reactive protein: risk marker or mediator in atherothrombosis? Hypertension 2004; 44(1):6-11.

10. Blann AD, Lip GY, McCollum CN. Changes in von Willebrand factor and soluble ICAM, but not soluble VCAM, soluble E selectin or soluble thrombomodulin, reflect the natural history of the progression of atherosclerosis. Atherosclerosis 2002; 165(2):389-391.

11. Chin BS, Chung NA, Gibbs CR, Blann AD, Lip GY. Vascular endothelial growth factor and soluble P-selectin in acute and chronic congestive heart failure. Am J Cardiol 2002; 90(11):1258-1260.

12. Francisco G, Hernandez C, Simo R. Serum markers of vascular inflammation in dyslipemia. Clin Chim Acta 2006; 369(1):1-16.

13. Blann AD, Belgore FM, Constans J, Conri C, Lip GY. Plasma vascular endothelial growth factor and its receptor Flt-1 in patients with hyperlipidemia and atherosclerosis and the effects of fluvastatin or fenofibrate. Am J Cardiol 2001; 87(10):1160-1163.

14. Blann AD, Belgore FM, McCollum CN, Silverman S, Lip PL, Lip GY. Vascular endothelial growth factor and its receptor, Flt-1, in the plasma of patients with coronary or peripheral atherosclerosis, or Type II diabetes. Clin Sci (Lond) 2002; 102(2):187-194.

15. Schachinger V, Zeiher AM. Atherogenesis-recent insights into basic mechanisms and their clinical impact. Nephrol Dial Transplant 2002; 17(12):2055-2064.

16. Kleinbongard P, Dejam A, Lauer T, Jax T, Kerber S, Gharini P et al. Plasma nitrite concentrations reflect the degree of endothelial dysfunction in humans. Free Radic Biol Med 2006; 40(2):295302.

17. Heiss C, Lauer T, Dejam A, Kleinbongard P, Hamada S, Rassaf $\mathrm{T}$ et al. Plasma nitroso compounds are decreased in patients with endothelial dysfunction. J Am Coll Cardiol 2006; 47(3):573-579.

18. Zachary I, Mathur A, Yla-Herttuala S, Martin J. Vascular protection: A novel nonangiogenic cardiovascular role for vascular endothelial growth factor. Arterioscler Thromb Vasc Biol 2000; 20(6):1512-1520.

19. Inoue M, Itoh H, Ueda M, Naruko T, Kojima A, Komatsu R et al. Vascular endothelial growth factor (VEGF) expression in human coronary atherosclerotic lesions: possible pathophysiological significance of VEGF in progression of atherosclerosis. Circulation 1998; 98(20):2108-2116.

20. Ramos MA, Kuzuya M, Esaki T, Miura S, Satake S, Asai T et al. Induction of macrophage VEGF in response to oxidized LDL and VEGF accumulation in human atherosclerotic lesions. Arterioscler Thromb Vasc Biol 1998; 18(7):1188-1196.

21. Yanni AE. The laboratory rabbit: an animal model of atherosclerosis research. Lab Anim 2004; 38(3):246-256.
22. Bocan TM, Mueller SB, Mazur MJ, Uhlendorf PD, Brown EQ, Kieft KA. The relationship between the degree of dietary-induced hypercholesterolemia in the rabbit and atherosclerotic lesion formation. Atherosclerosis 1993; 102(1):9-22.

23. Hernández LR, Lundberg U, Arocha-Pinango CL. Experimental thrombosis I: relation with fibrinogen and other haemostatic parameters. Thromb Res 2000; 99(3):295-305.

24. Jialal I, Devaraj S, Singh U. Sources of CRP in atherosclerotic lesions. Am J Pathol 2006; 168(3):1054-1055.

25. Sun H, Koike T, Ichikawa T, Hatakeyama K, Shiomi M, Zhang B et al. C-reactive protein in atherosclerotic lesions: its origin and pathophysiological significance. Am J Pathol 2005; 167(4):11391148.

26. Minor RL, Jr., Myers PR, Guerra R, Jr., Bates JN, Harrison DG. Diet-induced atherosclerosis increases the release of nitrogen oxides from rabbit aorta. J Clin Invest 1990; 86(6):2109-2116.

27. Moroe $\mathrm{H}$, Honda $\mathrm{H}$. Comparison of endothelial function in the carotid artery between normal and short-term hypercholesterolemic rabbits. Comp Biochem Physiol C Toxicol Pharmacol 2006; 144(2):197-203.

28. Nematbakhsh M,Hayat-Davoodi P,Rajabi P,Samarian SH.The effect of sterogen on endothelial permeability of aorta and the level of serum nitrite concentration in cholesterol-fed ovariectomized rabbit. Iran. Biomed J 2002; 6(2-3):77-82

29. Ferlito S, Gallina M, Catassi S, Bisicchia A, Di Salvo MM. Nitrite plasma levels in normolipemic and hypercholesterolemic patients with peripheral occlusive arteriopathy. Panminerva Med 1999; 41(4):307-309.

30. Tang FT, Qian ZY, Liu PQ, Zheng SG, He SY, Bao LP, Huang HQ. Crocetin improves endothelium-dependent relaxation of thoracic aorta in hypercholesterolemic rabbit by increasing eNOS activity. Biochem Pharmacol 2006; 72(5):558-65.

31. Cai H, Harrison DG. Endothelial dysfunction in cardiovascular diseases: the role of oxidant stress. Circ Res 2000; 87(10):840844

32. Minor RL, Myers PR, Guerra R, Bates JN, Harrison DG. Dietinduced atherosclerosis increases the release of nitrogen oxides from rabbit aorta. J Clin Invest 1990; 86:2109-2116.

33. Kanazawa K, Kawashima S, Mikami S, Miwa Y, Hirata K, Suematsu M, Hayashi Y, Itoh H, Yokoyama M. Endothelial constitutive nitric oxide synthase protein and mRNA increased in rabbit atherosclerotic aorta despite impaired endothelium-dependent vascular relaxation. Am J Pathol 1996; 148(6):1949-56.

34. Wilcox JN, Subramanian RR, Sundell CL, Tracey WR, Pollock JS,Harrison DG,Marsden PA. Expression of multiple isoforms of nitric oxide synthase in normal and atherosclerotic vessels. Arterioscler Thromb Vasc Biol 1997; 17(11):2479-88.

35. Verbeuren TJ, Bonhomme E, Laubie M, Simonet S. Evidence for induction of nonendothelial NO synthase in aortas of cholesterolfed rabbits. J Cardiovasc Pharmacol 1993; 21:841-845.

36. Andersen MR, Stender S. Endothelial nitric oxide synthase activity in aorta of normocholesterolemic rabbits: regional variation and the effect of estrogen. Cardiovasc Res 2000; 47(1):192-9. 